\title{
Les pouvoirs à l'hôpital : les relations entre médecins et directeurs
}

Alain Heriaud et Jean Arnautou

\section{(2) OpenEdition}

12 Journals

Édition électronique

URL : http://journals.openedition.org/communicationorganisation/3000

DOI : 10.4000/communicationorganisation.3000

ISSN : $1775-3546$

Éditeur

Presses universitaires de Bordeaux

Édition imprimée

Date de publication : 1 mai 1994

ISSN : 1168-5549

Référence électronique

Alain Heriaud et Jean Arnautou, "Les pouvoirs à l'hôpital : les relations entre médecins et directeurs », Communication et organisation [En ligne], HS N¹ | 1994, mis en ligne le 27 mars 2012, consulté le 01 mai 2019. URL : http://journals.openedition.org/communicationorganisation/3000 ; DOI : 10.4000/ communicationorganisation.3000

Ce document a été généré automatiquement le 1 mai 2019.

(c) Presses universitaires de Bordeaux 


\title{
Les pouvoirs à l'hôpital : les relations entre médecins et directeurs
}

\author{
Alain Heriaud et Jean Arnautou
}

1 Alain HERIAUD : Le thème qui nous a été proposé était «le pouvoir du corps médical et le pouvoir administratif ». Quand on me l'a proposé, j'ai répondu j'essayerais de voir parmi mes chefs de bureau ou mes adjoints si quelqu'un était disponible ce jour-là ; quant à moi je ne viendrais pas parce que je ne me considère pas dans cette catégorie (les administratifs). Je crois qu'il existe une différence de nature qu'il faudrait tout de suite poser. Le directeur d'hôpital à cet égard ne doit pas, mais peut-être est-ce sa faute, réduire son rôle et sa mission à faire de l'administratif. Son rôle est celui de diriger, comme tout dirigeant d'une entreprise. Or à vrai dire il n'est pas médecin. Mais le PDG qui vient de prendre la tête d'IBM vient de l'agro-alimentaire. Et dans un interview récent, il disait qu'il croyait bien que ses enfants, peut-être, avaient un microordinateur mais qu'il ne s'en était jamais préoccupé et qu'a priori, l'informatique n'était pas sa tasse de thé. Je crois savoir d'ailleurs qu'une actualité récente nous a monté que le PDG d'Air France n'était pas pilote de l'air, ce qui ne l'empêche pas de mener un certain nombre d'actions pour gérer son établissement. Donc, je dirais que le directeur d'hôpital se trouve à la tête d'une institution hospitalière dont il essaie avec les autres acteurs et dans un cadre communicant de faire qu'il réponde aux besoins pour lesquels il est fait, c'est-à-dire apporter les meilleurs soins à la population qu'il administre.

2 J'avais prévu de faire quelque chose de très didactique et puis à la fois, pressé par le temps et souhaitant peut-être que le débat avec la salle soit plus enrichissant que des discours trop académiques que nous pourrions tenir, je vais donc délibérément laisser de côté les propos que j'avais prévu de tenir. Mais je voudrais quand même jeter un petit coup d'œil en arrière parce que je crois que ce n'est pas inutile. Dans cette salle nous avons la chance aujourd'hui d'avoir une population qui n'est pas forcément très avertie $\mathrm{du}$ fonctionnement profond, intime de l'hôpital. Je suis même tenté de dire que les journées que nous connaissons aujourd'hui, il y a quelques années, personne n'aurait eu 
l'idée de les mettre en œuvre. C'est donc, qu'il y a eu une évolution et les propos de Mme Marx étaient parfois me semble-t-il un peu pessimistes quant à l'organisation actuelle de l'hôpital. J'en prendrai un petit peu le contre-pied. Je suis convaincu qu'énormément de choses s'y sont faites, si on essaie de faire un rapide historique. Je me servirais de trois dates un peu clés : avant les années 60, la période 60-80 et depuis.

Avant les années 60, l'hôpital qu'est-ce-que c'est?

L'hôpital, est une structure dans laquelle intervient un certain nombre de médecins, tous à temps partiel et qui considèrent que leur activité à l'hôpital est une activité, je dirais, accessoire. Donc, ils s'impliquent peu dans le fonctionnement de la structure si ce n'est pour venir y soigner individuellement un certain nombre de malades, ce qui est tout autre chose que de s'intégrer dans une problématique institutionnelle. Le Directeur de l'hôpital à l'époque, n'est pas formé la plupart du temps, si ce n'est qu'il a une certaine formation mais il n'a certainement pas la formation adéquate pour la gestion de l'hôpital en tant que tel.

5 Il a des pouvoirs extrêmement limités, partagés entre un pouvoir local, municipal où le maire de la commune est le personnage central qui recrute, bon bref un ensemble très complexe et en tous les cas, où de toute évidence les structures manquent de cohérence et de cohésion. Cela fonctionne malgré tout, on soigne tant bien que mal, pas toujours très bien quand même.

6 Et puis arrive la période que j'ai qualifiée de 60-80. Ce sont les vingt années que l'on peut considérer comme étant sans doute les plus actives à tous égards et où le développement de l'hôpital a été tout à fait fantastique, en terme de structure j'entends, en terme de développement quantitatif peut-être plus que qualitatif au sens relationnel et communicant, sujet qui est au cœur de nos débats de ces journées. En effet, c'est à partir de ces années, 1958 pour donner des dates d'ordre réglementaire et législatif mais ça n'a qu'une importance tout à fait anecdotique, que les médecins précisément vont devenir des acteurs de l'hôpital à part entière puisqu'ils vont consacrer la totalité de leur temps à l'hôpital. Parallèlement, les directeurs d'hôpitaux reçoivent désormais une formation spécifique distribuée par une école nationale, dites École Nationale de Santé Publique qui va former tous les ans, un bataillon de futurs cadres de direction hospitalière. Donc, on voit le paysage se former. Mais l'hôpital n'est pas exclu du monde qui l'environne, l'hôpital baigne complètement dans la santé. Et nous sommes à cette époque là, dans une société en forte expansion, où le chômage est résiduel et où les ressources de santé c'està-dire les ressources qui proviennent des organismes d'assurance maladie sont au beau fixe. Donc, dans cette période 60-80, la structure de direction de l'hôpital et la structure médicale prennent forme, au sein de l'institution et le font sans véritable conflit. Pourquoi ?... eh bien parce qu'il n'est pas obligatoire de communiquer lorsque tout va bien, le médecin soigne lorsqu'il a besoin d'un certain nombre de personnel. Je caricature un peu, c'est pour faire court. Le directeur de son côté est tout à fait heureux de pouvoir satisfaire non seulement les besoins que lui exprime le médecin, mais de façon plus générale les besoins que lui expriment la population. Plus l'hôpital est beau, plus il est grand et plus tout le monde est satisfait y compris le maire qui regarde d'un œil jaloux grandir de façon exponentielle la courbe des salariés de l'hôpital qui sont autant d'électeurs en puissance. Donc tout va pour le mieux dans le meilleur des mondes pendant cette période. Est-ce qu'il y a vraiment communication, je serais tenté de dire pas vraiment, le directeur et le médecin jouent leur rôle, mais jouent chacun leur partition. 
7 Et arrive la période 80 où là, les choses changent. Le contexte économique devient perturbé, la visibilité est réduite. Je ferai référence à un livre d'Hervé SERIEX « Le Bing Bang des Organisations ", qui décrit une déstabilisation relativement complète de la société en général, dans le monde industriel et dans les pays industrialisés. L'hôpital n'échappe pas à cette logique, si tant est qu'il y ait logique et on parle tout d'un coup de crise. Personnellement, je n'évoquerai pas le terme de crise parce que je pense que la situation de crise est une situation provisoire momentanée alors que ce en quoi nous sommes entrés est davantage une phase de changement qu'une phase de crise. L'hôpital se trouve sous les feux de l'actualité : l'hôpital dépensier, l'hôpital gaspilleur, l'hôpital fossoyeur des ressources de l'assurance maladie dit-on dans un certain nombre de périodiques. Les médias aiment. On faisait ce matin référence aux derniers rapports de l'IGAS qui traitaient effectivement des problèmes à la fois de la sécurité sociale et de l'hôpital. Un grand quotidien local qui reparait aujourd'hui, je ne citerai pas son nom, titrait « absence de sécurité à l'hôpital » sur cinq colonnes, alors que le contenu était sans doute plus nuancé. Mais cela fait recette parce qu'on aime bien lancer le débat sur ce thème. L'hôpital se trouve ainsi au carrefour de nombreux conflits. Véritablement il va falloir apprendre une nouvelle façon de gérer qui associe le corps médical et le corps de direction. Je dirais que, qu'on le veuille ou pas, il faut apprendre à travailler ensemble. Un certain nombre ont appris depuis un certain temps, mais je crois qu'aujourd'hui ceci est indispensable. On doit sortir des vieilles idées, idées reçues qui faisaient dire au corps médical : cette administration n'y connaît rien et ne voit l'hôpital que par le petit bout de la lorgnette de sa comptabilité. Et le directeur en face disant: de toute manière le médecin est complètement incompétent, il ne comprend que le « toujours plus » et il n'est absolument pas intégré dans la dynamique générale de l'institution et sa problématique est une problématique de paillasson qui se limite aux bornes étriqués de son service, quand ce n'est pas de sa propre personne.

8 Je crois qu'il faut sortir de ce type de relations qui effectivement ont eu cours pendant des années, pour ne pas dire des décennies et partir sur de nouvelles bases. Je m'arrête là, et je laisse la parole à mon ami Jean ARNAUTOU.

9 Jean ARNAUTOU : Merci Alain, bonjour à tous. Alors, il vient de le dire, nous sommes amis et ça fausse un peu peut-être le débat, si débat il doit y avoir. Notre amitié a quelque chose à voir avec ce qui nous réunit. Je remercie Alain HERIAUD parce qu'il m'a demandé de venir partager le temps de tribune qui lui est imparti. Notre amitié, elle s'est bâtie précisément sur la nécessité, sur l'impérieuse nécessité de trouver, dans un contexte qui, même s'il remonte à une vingtaine d'années n'était finalement pas très loin du contexte actuel, l'impérieuse nécessité d'ouvrir ou de maintenir ouvert des canaux de communication entre ces gens que l'évolution historique qu'il a décrite vont pratiquement condamner au conflit. Vous avez titré, je parle à l'organisatrice sur les pouvoirs à l'hôpital. C'est un sujet bateau dans notre milieu, peut-être pas dans le vôtre. Donc, on évitera d'y tomber, car l'approche du problème en terme de pouvoir dans ce genre d'enceinte n'est pas très payante. Je préfèrerais, si vous voulez, dire que la nature et, ce n'est pas simplement faire référence à un sujet philosophique et classique en période de baccalauréat, la nature du pouvoir dépend tellement des devoirs qui s'imposent ou qui devraient s'imposer aux acteurs qu'il vaut mieux se concentrer sur ce devoir auquel le directeur d'établissement obéit ou croit devoir obéir : le devoir d'intérêt collectif de l'institution. Cela va le conduire moralement à souhaiter que l'institution ne tombe pas dans la faillite financière. Il faut s'intéresser au devoir auquel le médecin obéit 
ou croit devoir obéir, qui est le devoir de respecter l'intérêt du malade et d'éviter cette faillite morale qui a conduit notre malheureux collègue GARETTA, par exemple accessoirement, je dirais à Frennes, mais plus gravement à enfreindre, vous le savez très bien une vieille règle plus que déontologique. Alors, c'est ça le conflit, pour lequel nous espérons que la communication va être un outil de résolution. Tout à l'heure Madame LAVILLE a très justement employé le mot distorsion, Mme LAVILLE est le médecin, je le rappelle responsable du service de la médecine du travail au CHU de Bordeaux. Elle a parlé de distorsion. Il est évident que si nous n'arrivions pas dans l'institution hospitalière à faire l'économie à chaque fois que c'est possible de ces conflits entre des impératifs catégoriques dictés par les devoirs éventuellement antagonistes, alors il y aurait risque majeur de distorsion de l'éthique des acteurs et cela peut se traduire par des lombalgies, non seulement pour l'aide soignant, mais aussi pour le directeur général. À terme, cela nous mènerait à un hôpital qui serait, je dirais pour les médecins, qui sont dans la salle entre l'hôpital schizophrène et l'hôpital auto-immun dans les maladies autoimmunes. Notre propre organisme se prend pour cible de ses propres agents destructeurs et il se détruit lui-même. On peut imaginer un hôpital en poussant évidemment les hypothèses d'école à leur extrême, un hôpital où les acteurs s'entre-dévorent parce qu'ils n'ont pas été capables de résoudre leurs conflits. Alors, pour ceux qui sont dans la salle, qui ne sont pas du milieu hospitalier cela peut paraitre un petit peu effrayant. Ils peuvent se dire « bon! on n'ira pas à l'hôpital public, on ira à l'hôpital privé parce que là-bas, il n'y a pas de problème d'argent du moins en France. Ils auraient tort car cela se passe exactement pareil dans les deux secteurs de la médecine hospitalière publique ou privée et c'est inévitable. C'est inévitable, parce que ce souci étroit, mesquin du médecin comme le caricaturait un instant Alain HERIAUD, d'avoir tout le temps et toujours plus, pour son malade qui est là. Ce souci, il ne va pas diminuer. Par ailleurs, le souci du Directeur de faire en sorte que les comptes soient à peu près équilibrés à la fin de l'exercice ne diminuera pas non plus. Dans un contexte où on nous a dit pendant vingt ans que la santé ou le souci de la santé, était pratiquement la contre productivité pour une économie moderne, bien! ce souci ne va pas diminuer. À ce propos, justement et après, j'ai pratiquement fini, je rends la parole à Alain HERIAUD, juste un dernier mot.

Dans le hall, vous avez une superbe carte que je connais depuis un an qui a été faite par la région Aquitaine et qui est la carte économique de l'Aquitaine. Beau pays, dont chacun connaît la richesse des sources minières, l'industrie en pleine expansion avec un taux de chômage sans doute le plus faible de l'hexagone. Vous m'avez compris, les non-aquitains peuvent savoir qu'on n'est vraiment pas très fort ni pour les ressources minières ni pour l'industrie lourde ou légère et quant au chômage, il ne doit pas être non plus particulièrement brillant. Regardez en passant, la santé, qui est le secteur $n^{\circ} 1$ de l'emploi en Aquitaine, par le biais de ce que les économistes appellent le coefficient multiplicateur-secteur permettant d'alimenter tout un tas de gens dans le secondaire et dans le tertiaire. À supposer que la santé soit primaire, la santé n'existe pas. Monsieur LEFEVRE, par exemple chef de l'entreprise la plus importante de la région Aquitaine, n'existe pas sur ce tableau économique, ceci juste pour vous dire qu'il faut prendre garde dans la résolution de nos conflits, de bien savoir où se trouvent les véritables frontières entre les impératifs du médecin et les impératifs du Directeur. J'en reviens aux gestionnaires, je n'aime pas employer ce mot quand nous sommes dans ce type de rencontre, nous parlons entre directeurs investis d'un pouvoir qui est très supérieur au pouvoir de gestionnaire. Il faudra se souvenir que dans nos débats lorsque nous arriverons aux frontières, que les intérêts économiques d'un pays moderne post- 
industriel passent aussi par la nécessité de maintenir opérant un système sanitaire et que ceci n'est pas productif.

11 Alain HERIAUD : Je voudrais donc prendre la balle au bond, pour revenir sur cette notion. C'est vrai que l'hôpital, je le disais tout à l'heure, et Jean ARNAUTOU vient de le rappeler, est souvent vu par le malade. Il espère, il a raison la plupart du temps d'espérer que la qualité des soins qui lui seront prodigués seront du plus haut niveau. Le problème est que le secteur hospitalier est un secteur très sensible. L'opinion de l'individu est différente selon qu'il est malade ou bien portant, dans un lot d'hospitalisés ou cotisant à la sécurité sociale. Et je crois que trop souvent, sans doute parce que nous sommes de mauvais communicants ou communicateurs, l'image de l'hôpital dans la population bien portante, celle qui regarde la télévision, qui lit le journal, qui vit normalement, est une image négative, les scandales divers les problèmes de financement font effectivement la une des journaux.. On parle des choses qui vont mal et on laisse le reste dans l'ombre. C'est dommage, et pour reprendre le mot de Jean ARNAUTOU, je crois qu'effectivement, l'importance économique des structures hospitalières n'était pas suffisamment mise en exergue jusqu'à des dates récentes où, sous prétexte ou en raison du projet de loi sur l'aménagement du territoire, on s'aperçoit tout à coup que l'hôpital n'est pas simplement un dépensier, n'est pas simplement fossoyeur de la sécurité sociale, mais qu'il revêt aussi une importance économique. Et c'est vrai, qu'il est tout à fait dommage que lorsque l'on présente une carte économique d'une région, le secteur hospitalier n'apparaisse pas. Pour ceux qui ne sont pas du milieu hospitalier, je voudrais préciser en effet que par exemple, le CHU de Bordeaux, est une entreprise forte de plus de 10000 salariés, ce qui signifie qu'elle fait vivre à minima 30000 personnes. C'est une entreprise dont le budget de fonctionnement annuel s'élève à plus de 3 milliards de francs. C'est donc, une entreprise qui déverse dans les tissus économiques locaux et régionaux, des sommes non négligeables qui permettent de faire vivre d'autres structures, d'autres entreprises, d'autres commerces. Soyez bien convaincu du fait que ces entreprises-là en sont complètement conscientes. Lorsque certains habitués de nos marchés se voient par le jeu de la libre concurrence privés du marché de l'hôpital, c'est souvent pour ces entreprises non seulement une déception, mais parfois un risque majeure de disparition. Alors une fois qu'on a dit ça, et que nous sommes d'accord, en deux mots, comment mettons nous en place la communication?

12 Je ne ferai que deux flashs, il y a une communication institutionnelle qui existe, dont il a déjà été question ce matin. On vous a parlé de la commission des soins infirmiers, on vous a parlé du conseil d'administration, on vous a parlé du comité technique d'établissement. Je pourrais rajouter une pierre importante qui est la commission médicale d'établissement. Pour ceux d'entre vous qui ne le sauraient pas c'est en fait l'assemblée constitutive du corps médical de l'hôpital dans laquelle siège toute ou partie des médecins de l'hôpital selon la dimension de la structure. Au CHU de Bordeaux, nous avons pratiquement 500 médecins titulaires et, je ne parle pas des internes, des chefs de cliniques, des assistants, 500 médecins titulaires permanents. Il est bien évident que les 500 ne siègent pas car ce serait un parlement dans lequel sans doute le caractère artificiel des débats serait plus imposant que l'efficacité. Mais nous avons une commission médicale d'établissement qui est un lieu d'échange et de dialogue bien entendu. Aujourd'hui à Bordeaux, nous avons créé une quinzaine de commissions spécifiques ciblées orientées, vers un certain nombre de problématiques de façon à ce que le corps médical et la direction dialoguent, tentent de faire des choix, puisque nous en sommes 
bien aujourd'hui à une politique de choix. Le "toujours plus » est terminé, et je pense pour longtemps. Les choix que nous faisons dans un contexte de diminution de moyens doivent être les mieux partagés possible. Et pour que ces choix soient les mieux partagés possible nous avons créé un certain nombre de commissions qui travaillent sur l'informatique, sur les équipements, sur la recherche à l'hôpital, sur la formation des personnels médicaux, problème important qui a été évoqué, car là effectivement nous touchons à l'éthique. Un hôpital pour des raisons économiques va devoir parfois réduire un certain nombre de moyens, voir s'opposer à certains traitements, non urgents, étant entendu que notre vocation et notre obligation nous imposent bien entendu, hors de toute considération économique d'accueillir tous les malades qui ont besoin en urgence de soins qui doivent leur être conférés en milieu hospitalier. En dehors de cet aspect d'urgence, nous avons créé ce que nous avons appelé une commission de la décision médicale qui rassemble cadres de direction et médecins pour un certain nombre de problèmes que nous rencontrons dans ce domaine de la non urgence. Un exemple, et je m'arrêterai pour repasser la parole à Jean ARNAUTOU. Prenons le cas d'un malade, encore une fois non urgent qui nécessite une intervention chirurgicale; ce malade se trouve être malheureusement un hémophile, on sait que l'intervention chirurgicale est nécessaire et que seul l'hôpital public l'acceptera, souvent le CHU, à la fois pour des considérations de technologie, de compétence, mais aussi de finances. Il faut que vous sachiez que dans un cas comme celui-ci, il peut se faire qu'un seul malade, je dis bien un seul malade pour une intervention chirurgicale, nécessite jusqu'à 3 voire 4 millions de francs en produits sanguins et dérivés divers. Eh bien, une commission se réunit, afin d'évoquer cette question, afin de voir comment économiquement, éthiquement, médicalement, on va pouvoir aboutir à une décision concertée dans l'espace et dans le temps. Et ça peut aller jusqu'à, effectivement, des moyens spécifiques et exceptionnels revendiqués auprès des autorités de tutelle. Cela peut passer par des choix qui feront que certaines choses qui étaient prévues ne se feront pas pour privilégier ceci, mais ceci se fait dans un climat concerté car aujourd'hui, je reviens sur ce que je vous disais, en commençant mon propos, cela me semble incontournable. Nous devons passer à une logique solidaire. Nous ne sortirons pas de cette logique, je crois qu'il faut que chacun en soit intimement convaincu. Et je crois aussi qu'il faut passer d'une logique d'addition, à une logique de multiplication. Il ne suffit pas d'additionner les compétences à l'hôpital, il faut les mêler, il faut les multiplier car si en arithmétique élémentaire 10 plus 10 font 20 ; 10 multiplié par 10 font 100 . Je crois que cette logique arithmétique, on doit la retrouver au niveau de notre institution hospitalière.

13 Jean ARNAUTOU : Je vais donc conclure après quoi la parole sera évidemment et nous le souhaitons en tout cas je pense, à tous ceux qui se taisent depuis longtemps. Les anglais aiment bien dire quand on ne veut pas résoudre un problème, on crée une commission, et ça n'a pas manqué au CHU de Bordeaux. On a donc créé une commission dont celle de la décision médicale. À l'hôpital d'Agen bien sûr, on a fait pareil, mais je ne crois pas que ni à Agen ni à Bordeaux, on ne souhaite pas résoudre les problèmes. C'est pour ça que j'aimerais revenir sur ce pourquoi on est copain avec HERIAUD. En travaillant ensemble dans le même hôpital, il y a une quinzaine d'années, on était arrivé à la conclusion, assez vite, qu'il fallait absolument trouver le moyen de s'entendre sous peine de très graves dérèglements. Et on a donc présenté une proposition d'organisation de la décision hospitalière qui passe par cette nécessité de la concertation et donc de la communication. Et on l'a présenté dans une réunion à laquelle participaient des personnes bien connues des deux ténors que j'ai cité tout à l'heure, le directeur des hôpitaux à l'époque et 
Philippe CADENE qui était le patron du monde hospitalier français. Et l'un et l'autre, avaient souhaité qu'on aille un peu plus loin et ça a donné lieu au mois de décembre 1981 à Paris, dans le cadre des Assises Hospitalières, à une présentation qu'on a appelé la Direction participative à l'hôpital. La direction participative par objectif : la DPO, est une technique de management déjà maintenant ancienne. À l'époque elle l'était un peu moins, appliquée au départ dans le tissu industriel ou libéral, certains pensaient qu'elle pouvait être adaptée à l'hôpital public. Mais l'époque était mal choisie. En décembre 1981, c'était l'entrée du monde hospitalier français en PMSI, peu importe pour ceux qui ne sont pas du monde hospitalier ici, la signification exacte de ces initiales. À l'époque, elle a signifié pour celui qui en était le promoteur un téléguidage de l'hôpital français par l'instance la plus centralisée c'est-à-dire par la direction des hôpitaux. Et ceux qui avaient la fibre hospitalière souhaitaient que l'organisation reste au moins en grande partie contrôlée par ses acteurs de terrain c'est-à-dire conserve son autonomie d'établissement. Donc, l'entrée en PMS1 a fait que la direction par objectif à l'hôpital a été longtemps repoussée. Elle n'était pas morte et depuis quelques mois démarrent en France deux expériences de gestion moderne des hôpitaux. L'une via le PMSI, l'expérience Languedoc Roussillon à laquelle j'ai eu le plaisir de participer, quoique n'étant pas un adepte de l'outil qui la soutend. L'autre est l'expérience de direction par objectif tel que la fédération hospitalière l'a développée dans ses hôpitaux. C'est donc un sujet d'actualité que nous traitons aujourd'hui mais il faut dire qu'il faut des commissions. Mais, j'ai une grande nouvelle pour ceux d'entre vous qui ne lisent pas dans le texte HABERMAS et APEL, philosophes allemands contemporains ou Paul RICOEUR philosophe français quasicontemporain, car j'ai appris grâce aux directeurs d'hôpitaux réunis à Evian il y a quinze jours, j'ai appris grâce à un professeur de philosophie à la Sorbonne, Simone PARNEX qu'en fait dès lors que vous réunissez des gens qui sont des experts concernés par le sujet sur lequel ils vont discuter, la décision à laquelle ils vont parvenir sous réserve qu'ils respectent chacun leur éthique personnelle et l'éthique de l'interlocuteur, sera une décision éthique.

L'éthique peut naître de la dialectique, si vous voulez pour résumer très sommairement. Cela m'a bouleversé parce que j'avais ainsi une base je dirais déontologique à la justification de ces multiples réunions auxquelles ceux qui sont du milieu hospitalier commencent à donner le nom de réunionite. Ils se demandent jusqu'à quand ils vont pouvoir les subir car c'est vrai que la loi de 91 pose la nécessité du projet d'établissement qui multiplie à l'envie les réunions. La réunion peut avoir cette vertu très particulière de déboucher sur une décision éthique. Mais encore une fois, il y a les présupposés. Il est important de ne pas les oublier : il faut le respect de sa propre éthique et le respect de l'éthique de l'interlocuteur. Alors, munis de cette dogmatique sur la nécessité de communiquer ensemble à travers ce travail de commission, afin de parvenir à dégager les objectifs prioritaires de l'institution, qu'est ce qu'on va essayer de faire à Bordeaux dans la commission de la décision médicale, à Agen dans la commission du projet et des objectifs? On va essayer de faire en sorte que ces conflits dont je disais tout à l'heure qu'ils étaient quasi inéluctables, que ces conflits soient prévenus, chaque fois que possible. C'est possible dans bien des cas d'ailleurs les professionnels de la communication ici le diraient mieux que moi. On essayera de les résoudre. Nous serons les sapeurs pompiers dans l'hôpital, je dis nous: le directeur, le président de la commission médicale, parce que la loi française a défini les rapports qui existent entre ces individus et puis les équipes qui sont autour d'eux. Nous serons les sapeurs pompiers, nous éteindrons tous les feux de brousse qui immanquablement commencent ici et là, et 
en bons sapeurs pompiers, nous saurons aussi jusqu'où peut aller le feu parce qu'il est bien évident que l'harmonie qui règne aujourd'hui à la tribune entre Alain HERIAUD et moi, ne règne que parce qu'on ne travaille pas dans un même hôpital. Si demain, on était dans la même boîte, on serait constamment plus ou moins en conflit, comme on doit le faire mais pas n'importe où, pas n'importe comment, et c'est pour ça que ces commissions sont très précieuses. Pour les hospitaliers, je dirais même pour les professionnels de la communication, elles permettent d'éviter que lors des réunions officielles, que sont la commission médicale, le comité technique d'établissement et le conseil d'administration, apparaisse l'image d'une direction d'hôpital schizophrène. Il faut donc que ces commissions préalables permettent d'éponger les conflits, permettent d'éteindre les incendies qui démarrent, de faire la part des choses, c'est-à-dire de savoir jusqu'où on peut aller dans le compromis sans aller dans la compromission. Je finis là-dessus, en rappelant, qu'en fait, l'éthique du directeur et l'éthique du docteur ne sont pas si opposées que ça. Prenons un médecin attaché à l'intérêt de son malade, comme Ulysse au mât de son bateau, dans ce cas je plains le directeur d'hôpital car sa propre éthique va souffrir très rapidement après qu'ait souffert l'éthique du médecin. Parce que s'il est devenu directeur d'hôpital, ce n'est pas tout à fait innocemment. Maintenant les directeurs d'hôpitaux sont entrés dans cette carrière, un peu peut-être comme on entre en religion. Quand à l'éthique du docteur si d'aventure, il a affaire à un directeur laxiste, qui fait que l'hôpital est en banqueroute à la fin de l'exercice, elle va sans doute assez vite en souffrir. Un autre directeur arrivera après, ou non, ce sera Monsieur le directeur régional de la santé, ou Monsieur le contrôleur des finances, qui viendra mettre de l'ordre. Donc en fait, nos intérêts mais au sens un peu noble du terme, nos intérêts sont donc sans doute assez proches les uns des autres. Nous avons donc tout intérêt à-ce que ces commissions intermédiaires fonctionnent et que les canaux de communication soient ouverts. Si on doit s'accrocher en commission médicale ou en commission des objectifs à Agen, peu importe mais quand on sortira on aura fait la paix.

Béatrice GALINON-MELENEC : Nous avons bien compris que ce duo était parfaitement orchestré. La mise en scène nous a permis d'entendre quelque chose de très brillant et nous vous en remercions. Permettez-moi de marquer une petite réserve par rapport à ce discours qui renvoie effectivement à la logique managériale comme vous l'avez rappelé souvent. La logique managériale n'est-elle pas une logique de verticalité de l'information?

16 Alain HERIAUD : Vous évoquez la verticalité, nous aujourd'hui nous sommes entrés complètement dans une logique de transversalité. Quand je dis une logique de transversalité, c'est que précisément dans notre vision de l'hôpital et je rejoindrai certains propos qui ont été tenus ce matin, on doit sortir, excusez-moi encore cette comparaison musicale, d'une logique du tuyau d'orgue pour passer à une logique de complémentarité à la fois dans les missions de chacun et dans la chaîne dans laquelle chacun doit se situer. Tous les acteurs de la chaîne s'inscrivent dans cette chaîne de transversalité des compétences afin que naisse une amélioration de la qualité du service rendu auprès de la population que vous desservez. Pour nous c'est clair, la verticalité non, la transversalité oui. Notre stratégie qui vise par exemple à confronter la problématique économique, la problématique médicale et la problématique éthique qui se présente dans un cas spécifique, montre que nous souhaitons travailler en transversalité et non pas en verticalité. Je suis convaincu que le processus de décision descendant fait partie de l'histoire et qu'on n'y reviendra pas. 
17 Prenons l'exemple de la CME, Commission Médicale d'Etablissement. Cet organe qui a une importance vitale pour l'hôpital, c'est un organe purement médical. Il regroupe les médecins et n'est pas un organe hiérarchiquement inféodé à la Direction de l'établissement. Il a toute liberté pour participer à la politique de l'établissement. Il donne des avis, il est consultatif mais c'est un lobby extrêmement puissant dans la définition de la politique de l'hôpital. En amont de cet organe qui risquerait, à cause de cette situation de lobby puissant de s'enferrer dans des positions parfois totalement déconnectées de la réalité hospitalière, ce que nous avons essayé de promouvoir à travers le mécanisme de direction participative par objectif, c'est l'émergence d'une commission transversale. Mais cette transversalité, je vous prie de croire que culturellement elle ne va pas de soi. La transversalité va très loin culturellement. À Agen, il y a douze personnes. Vous avez six médecins mais vous avez en face l'équipe de direction au complet incluant le directeur des soins infirmiers, l'ensemble des responsables des secteurs économiques, financiers, et on se parle sur un mode qui n'existait pas auparavant. Jusque-là, dans la CME on parlait en présence des cadres de l'hôpital mais on ne parlait pas avec eux. On se retrouvait au Conseil d'Agen et là le ton était encore complètement différent parce que les gens arrivaient avec des casquettes syndicales ou corporatives. Cette commission en amont n'est pas écrite dans la loi, elle est complètement officieuse et informelle. On y parle d'un tout autre ton, celui des gens qui, encore une fois, dans la transversalité s'efforcent de conjuguer les désirs d'absolu des uns et des autres et la nécessité faisant loi à la fin. Je vous assure ce n'est pas évident. Je vous assure que c'est révolutionnaire dans une entreprise telle que l'hôpital où s'exerce une profession - au rôle quasi sacerdotal et qui n'était pas jusqu'à présent très désireuse de le partager d'une manière ou d'une autre.

M. KENY, chirurgien orthopédiste à l'hôpital de Poissy en région parisienne : La difficulté de communication avec le corps médical n'a pas été beaucoup expliquée. Je pense qu'on a bien compris que la communication c'est l'écoute, c'est surtout passer du temps. Pour prendre l'exemple des infirmières, un cadre infirmier, une surveillante qui s'occupe de la communication et de l'encadrement laisse en partie tomber les soins et s'occupe plutôt de la communication. Il y a donc une contrainte de temps des médecins, assurer le bloc opératoire, assurer les consultations, assurer les visites, en plus passer du temps dans les réunions et faire de la communication. Je pense que la communication est indispensable mais il faudra de la part de l'administration trouver des vecteurs de communication adaptés à cette contrainte qui est réelle Deuxièmement, les problèmes de pouvoir ont été un peu esquivés au départ. Il y a un conflit de pouvoir à l'hôpital entre le corps médical et l'administration parce qu'il y a des contraintes budgétaires et donc des choix. Tout le problème est de savoir qui a le dernier mot pour faire le choix et c'est le cœur de ce conflit.

19 Alain HERIAUD : Sur la première de vos questions, je dirais que c'est un peu un faux débat. Il est évident qu'aujourd'hui, seules les entreprises communicantes ont pu affronter le choc économique dans les meilleures conditions. Il faut donc trouver des moyens, je suis d'accord avec vous, il ne faut pas que l'arbre du travail quotidien cache la forêt de l'ensemble des besoins de l'institution. Le deuxième problème est le problème des pouvoirs. Nous avons souhaité, Jean et moi, esquiver un petit peu ce débat parce qu'il ne nous paraissait pas forcément cadrer avec la ligne directrice, j'allais dire rédactionnelle de ces journées. Mais si je veux vous répondre de façon abrupte, il est difficile d'avoir une réponse manichéenne sur qui va avoir le dernier mot. Je ne crois pas qu'il faille justement poser le problème en ces termes. Par exemple, dans la commission de décision médicale 
que nous avons au CHU de Bordeaux, nous faisons en sorte que personne ne puisse revendiquer précisément ce dernier mot. Mais. Plus exactement la décision que l'on prend, on la prend ensemble. Le directeur reste et demeure le seul responsable in fine non seulement sur le plan économique mais aussi sur le plan juridique, seul représentant de son institution devant la loi et devant les tribunaux.

Jean ARNAUTOU : Je reviendrai sur la fin de ce que tu viens de dire Alain. Il peut arriver qu'il y ait désaccord mais il n'est pas question d'entrâner l'autre dans une voie qu'il ne veut pas suivre. Les conflits de pouvoir, je le répète, sont des conflits qui recouvrent des conflits de devoir. Il n'est pas question que votre éthique de chirurgien soit obérée par l'éthique du directeur et vice versa. À un moment donné, il reviendra au directeur de trancher et vous restez chirurgien. La décision est prise et supposons qu'elle concerne la fermeture de votre service pendant le mois d'août parce qu'après tout les interventions chirurgicales peuvent se faire dans la clinique du coin. C'est une problématique tout à fait envisageable dans l'hôpital public français actuel. Alors, on va fermer pendant quinze jours. Vous pouvez pour des raisons qui sont parfaitement valables ne pas souhaiter cela. La décision reviendra au directeur, il fermera le service. Vous gardez de puissants moyens de faire en sorte que cette décision, peut être, ne soit pas appliquée par exemple, ou bien soit appliquée partiellement, ou bien soit appliquée cette année mais que pour l'année prochaine on fasse tout pour qu'il n'y ai pas besoin qu'il la prenne. L'important, est que la communication entre vous et lui ne soit pas interrompue. C'est que chacun dise: "A partir de maintenant chacun va agir en fonction de ses obligations ». Nous reprendrons bien entendu le dialogue la minute d'après sur un autre sujet et la semaine d'après, peut être sur le même sujet dans une nouvelle donne. C'est cela qui est fondamental. Préserver la capacité de l'entreprise à maintenir un canal de communication ouvert quoiqu'il arrive.

M. LEFEVRE Directeur d'hôpital : Je serai très rapide, je pense que nous avons passé toute la matinée et même une partie d'hier dans le microcosme. C'était bien naturel d'ailleurs puisque nous nous y trouvons, malgré certains états d'âme, heureux. Mais quoiqu'il en soit, il est bien évident que le monde de la santé est légitimement inquiet et qu'il subit des actions extérieures que l'on peut qualifier de nuisibles. Il y a au niveau de la question de la santé en général une tendance à la bureaucratisation de son système de gestion. Je parle du système de gestion de la protection sociale au niveau national et nous constatons, à l'hôpital comme ailleurs, Michel CROZIER l'a fait depuis très longtemps, les signes d'une société bloquée. Il est certain que la loi de réforme hospitalière du 31 juillet 1991 a eu comme objectif d'engager au niveau de l'hôpital ce que j'appellerais un exercice de psychothérapie de groupe, car il importait de corriger justement toutes les critiques qui pouvaient être apportées par les divers groupes sociaux à l'intérieur de l'hôpital. M.ARNAUTOU et M. HERIAUD étaient chargés, ce matin, de se battre et ils ne se sont pas battus. On disait qu'il existait un pouvoir administratif et un pouvoir médical et, ensuite, il est apparu un troisième pouvoir, c'est le pouvoir infirmier. Par conséquent, il faut éviter de sombrer dans ce faux débat sachant d'ailleurs que l'autorité de tutelle, l'autorité ministérielle a très légitimement considéré d'ailleurs où il convenait de parler de gestion médicalisée de l'hôpital. Mais vous voyez tout de suite ce que peut contenir comme ambiguïté ce terme. Alors, au-delà de cette définition donnée au projet d'établissement qui est un exercice convivial mais un exercice de psychothérapie de groupe, il faut donner à ce projet l'importance qu'il mérite. Il est évident qu'il s'agit d'un projet stratégique et dont l'enjeu est extrêmement important. Quittons très rapidement l'hôpital pour considérer que, justement, cette loi de réforme hospitalière a prévu la mise 
en œuvre d'une planification régionale. Ce sont des termes brillants. Ce sont des termes apparemment très sérieux mais il faut être logique. La planification régionale en matière hospitalière doit porter sur deux secteurs: le secteur hospitalier public et le secteur hospitalier privé, notamment celui à but lucratif. Et chacun sait que les pouvoirs publics n'ont aucune action sur l'évolution même des capacités des établissements privés à but lucratif, que ceux-ci, d'ailleurs étant très actifs, ne perdent pas beaucoup de temps actuellement à se restructurer, ni à créer les évolutions nécessaires. Je crois qu'il faut en avoir conscience. Vous voyez par exemple, dans des grandes villes universitaires des cliniques de six cents lits qui commencent à apparaitre par regroupement et par restructuration. Ces établissements ont d'ailleurs des moyens de financement qui peuvent être mobilisés extrêmement rapidement Donc ceci veut dire que le secteur hospitalier public devient un secteur résiduel. C'est le secteur résiduel qui est le seul objet des contraintes et pressions des pouvoirs publics. Cela veut dire que cet enjeu qui conditionne réellement la vie future ou à moyen terme des établissements, est un problème extrêmement important. Il y en a un autre c'est que, bien sûr, on peut tenir compte de tous les états d'âme et des réflexions sans doute très justifiés de tels groupes professionnels au niveau de l'hôpital, mais il est bien certain qu'un projet d'établissement doit porter effectivement sur une stratégie médicale c'est-à-dire sur la définition d'objectifs médicaux et que ceci doit constituer la base essentielle de ce projet. Un récent rapport nous dit que l'hôpital doit répondre d'une part à la notion d'urgence, c'est-à-dire que tout hôpital qui ne disposera pas d'un service d'urgence agréé et qui ne disposera pas d'un plateau technique adapté est condamné à la mort. Il faut que le projet d'établissement ne soit pas l'expression d'un rêve mais qu'il colle avec les moyens financiers susceptibles de le mettre en œuvre. Par conséquent il faut que les hôpitaux, sous peine de disparaître, s'engagent. Cela signifie qu'il faut que le projet d'établissement soit un engagement, une motivation, une action dynamique. Il faut que, demain, apparaisse au niveau régional un véritable réseau hospitalier bien organisé et que l'hôpital ouvre même sa porte à la médecine libérale et aux établissements privés pour la desserte de son plateau technique, compte tenu d'ailleurs de l'importance des investissements qu'il doit générer. C'est pourquoi, je pense qu'il faut redonner au projet d'établissement sa réelle dimension et considérer qu'à ce titre, il s'agit même d'une opération de survie de l'établissement.

\section{AUTEURS}

\section{ALAIN HERIAUD}

Directeur Général Adjoint du CHU de Bordeaux

JEAN ARNAUTOU

Président du CME du CH d'Agen 